\title{
Integrating Complementary Tools For Depth Imaging
}

\author{
S. Jerry Kapoor \& Uwe K. Albertin \\ WesternGeco, 3600 Briarpark Ave., Houston , TX 77042
}

\begin{abstract}
Several field examples of Kirchhoff depth imaging, tomography, and wavefield-extrapolation imaging are presented. These examples illustrate how compensation for anelastic attenuation, multiple attenuation, tomography, and wavefield extrapolation can be used in a complementary fashion to provide improved interpretation and imaging in areas of significant geologic complexity.
\end{abstract}

\section{INTRODUCTION}

In the past several years, numerous successes in subsalt exploration have been obtained with imaging tools such as Kirchhoff migration. Recently, however, a number of advances have been made that improve depth imaging considerably in such areas. Multiple attenuation and compensation for anelastic absorption losses have optimized the clarity and frequency content of primary events in the migrated image. Tomography has produced more accurate velocity updates that take into account complex ray bending. Finally, wavefield-extrapolation migration has improved imaging in the vicinity of salt bodies and below rugose salt when compared with traditional Kirchhoff migration. Here we present several field examples from complex areas in the Gulf of Mexico that illustrate how a processing flow combining these techniques can significantly improve image fidelity.

\section{COMPENSATIONFORATTENUATIONINPROPAGATION AND MULTIPLE REMOVAL}

As sound passes through complex sediments and salt interfaces in the Gulf, significant mode conversion, reverberation, and attenuation occur. During wave propagation, geometric spreading and anelastic attenuation reduce the amplitude of primary events, while mode conversion and multiple reflections create coherent noise that should be removed for a proper image. As the structure becomes more complex, these effects often become more pronounced, and proper compensation becomes more critical.

An example of extreme sediment complexity, where both optimized multiple attenuation and optimized compensation for absorption losses had a significant impact, is shown in Figure 1. This Figure shows time and depth images obtained by migrating with the sediment velocity field only, prior to insertion of salt, which lies some distance away from this line. In this area, initial processing followed by Kirchhoff time migration revealed a highly complex sediment field, but the extreme complexity obscured the steep-dip sediment areas, as seen in Figure 1a. Following this initial processing we applied an optimized flow consisting of surface-related multiple attenuation (Dragoset et al, 2000) and optimized absorption compensation. The results are quite dramatic, as seen in Figure $1 \mathrm{~b}$, where sediment detail and vertical sediment faces are now visible deep into the section. This example illustrates the improvements possible through integration of multiple attenuation and proper anelastic absorption compensation in a standard Kirchhoff imaging workflow.

\section{TOMOGRAPHY AND WAVEFIELD EXTRAPOLATION MIGRATION}

In sediment areas of the Gulf, migration interval velocities are well described by continuous functions, with discontinuities occurring only at salt boundaries. In these areas, grid-based tomography is an effective tool for updating velocities (Woodward et al, 1998). Significantly flatter migrated gathers can often be obtained by using iterative grid tomography in which length scales of the velocity updates are progressively reduced for stability and convergence. For efficiency, we utilize autopicking of both hyperbolic and complex moveout on gathers based on energy semblance. We also make extensive use of autopicking of dip fields required in the ray-tracing portion of the tomographic backprojection.

One critical phase of tomographic updating in the Gulf occurs as rays hit salt boundaries. Assuming that complex moveout on migrated gathers is attributable entirely to sediment velocity errors, ray bending at salt boundaries will introduce instabilities and spurious velocity updates. For this reason we have implemented grid-based tomography with a hybrid model representation in which salt surfaces are embedded in the velocity model used by the ray tracer. In this way, it is possible to signal when a ray hits the salt interfaces, and to surgically remove it from the tomographic update procedure. This produces an update that is stable, and is indicative of actual sediment velocity variations. 
An example of tomographic updating in the Gulf in the area of Figure 1 is shown in Figure 2. Figure 2a shows the migrated gathers before the update, while Figure $2 b$ shows the results after the update. Examination of the final interval velocity showed that the grid tomography updated and localized the velocity variations in a manner consistent with the geology. As can be seen in the Figure, the gathers were significantly flattened.

In areas where salt complexity prevents Kirchhoff migration from adequately imaging gathers, the use of wavefield-extrapolation migration to produce angle gathers is

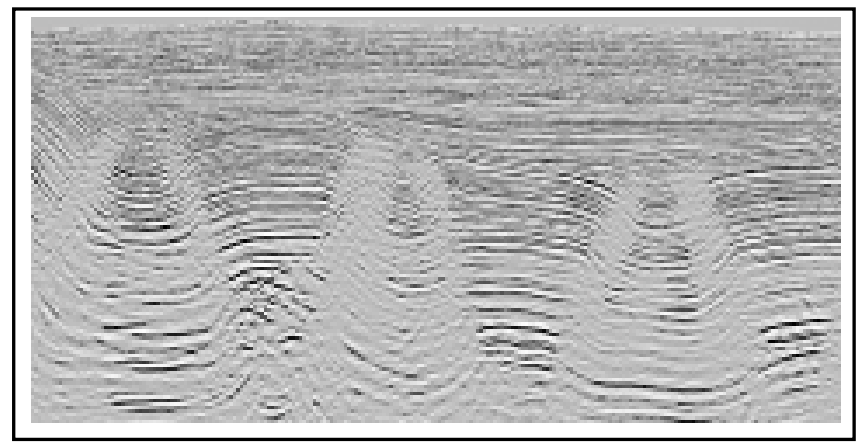

Figure 1a: Initial time imaging in an area of significant complexity in the Gulf of Mexico

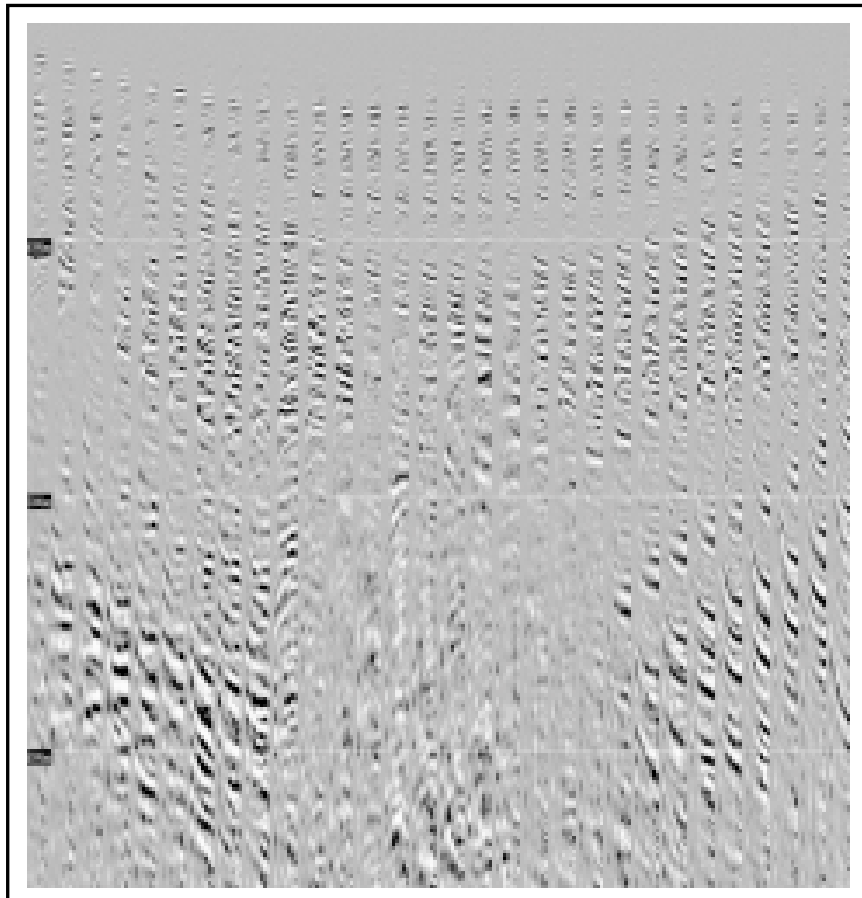

Figure2a-b: Kirchhoff migrated gathers prior to tomography (Figure 2a, left), and after tomography (Figure 2b, right). an effective alternative. An example of this is seen in Figures 3a-c. Figures 3a-b show a comparison of Kirchhoff and wavefield-extrapolation migration of a complex tabular salt structure. Under the salt nose, Kirchhoff migration has difficulty in imaging, while the structure in the wavefieldextrapolation migration is significantly improved. This improvement carries over to the wavefield-extrapolated angle gathers, seen in Figure 3c. Although one might expect that a ray-based velocity update technique that uses wavefieldextrapolated gathers would produce an update that is inconsistent, recent Kirchhoff migration results using

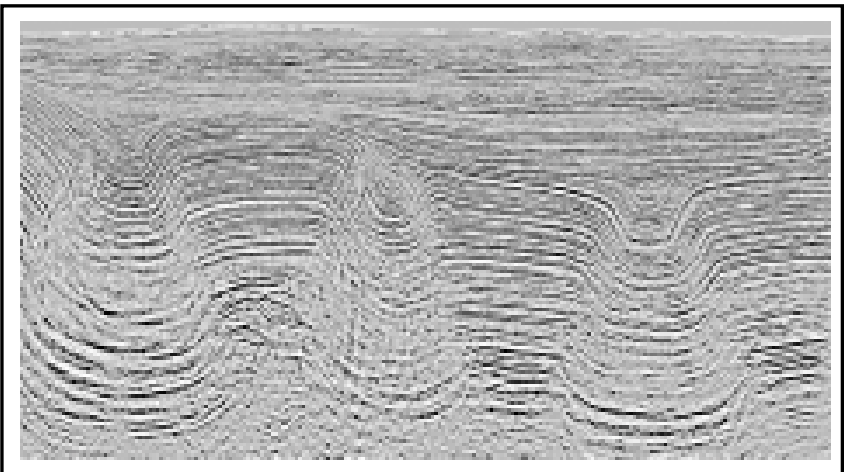

Figure 1b: Reprocessed depth-imaged data with absorption compensation and improved multiple attenuation.

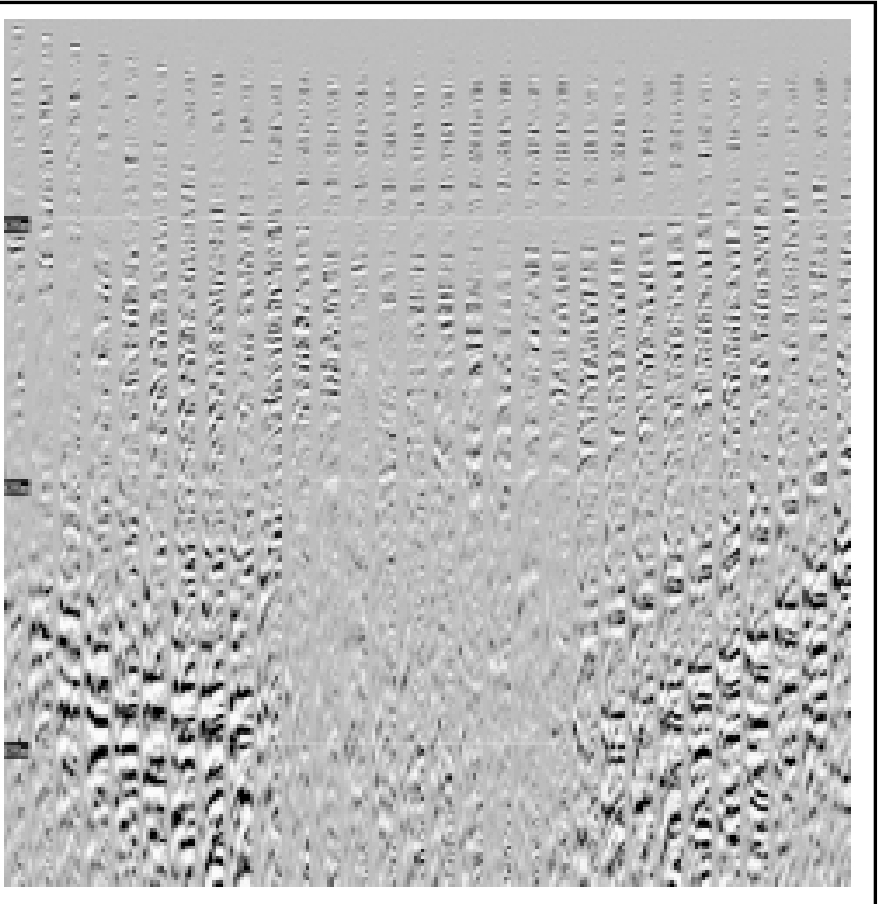




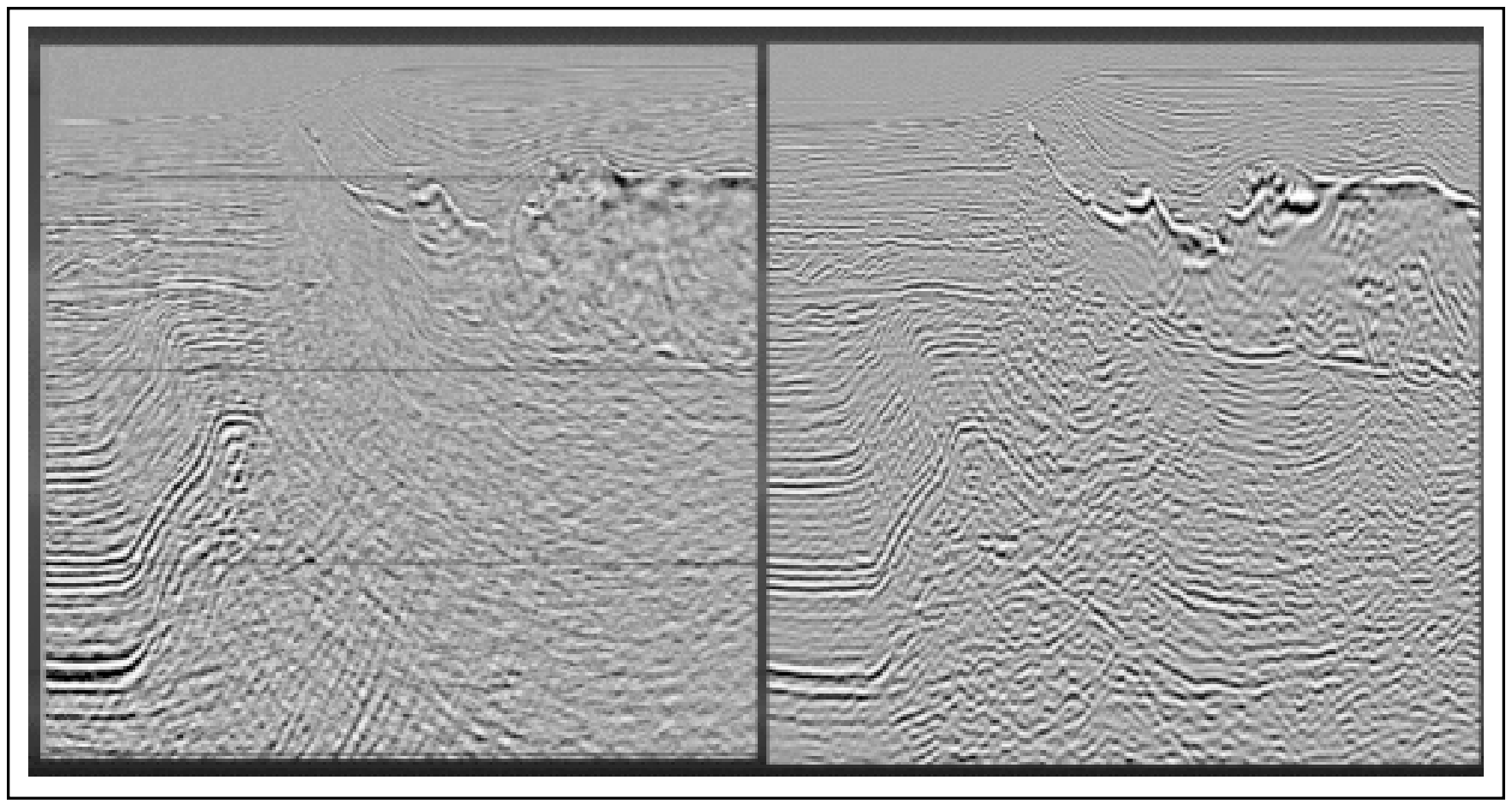

Figure 3a-b: Kirchhoff migration ( Figure 3a, left) and the corresponding wavefield extrapolation migration (Figure 3b, right).

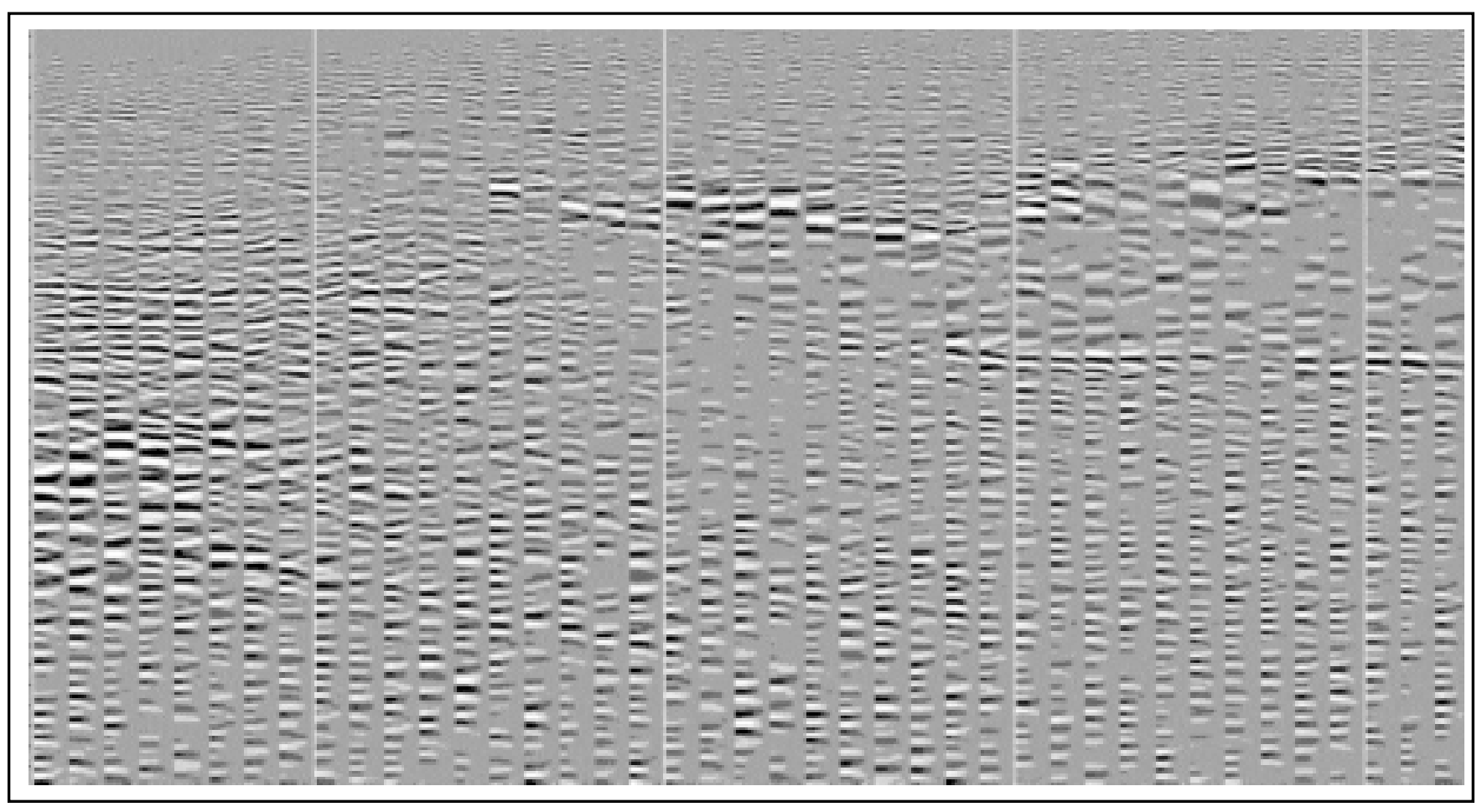

Figure 3c: Wavefield-extrapolated angle gathers in the same area as Figure 3a-b. 
amplitudes and phases with upward ray-shooting show improvement in image quality and handling of multi-pathing when compared with traditional Kirchhoff migration. Since tomography uses a similar upward ray shooting, and is primarily concerned with kinematics in updates of sediment velocities, it is plausible that tomography coupled with wavefieldextrapolated angle gathers will yield good results. Our investigation of these gathers and their use in tomographic updating is currently underway.

\section{CONCLUSION}

In summary, complementary use of proper compensation for attenuation, good multiple attenuation, and use of tomography and wavefield-extrapolation migration is an effective strategy for improving depth imaging in complex areas found in the Gulf of Mexico.

\section{ACKNOWLEDGEMENTS}

The authors would like to thank their colleagues at Shell for their help in obtaining some of the results shown here. The authors would like to thank WesternGeco for permission to publish these results.

\section{REFERENCES}

Dragoset, B., Bell, J., Pattberg, D., Gulunay, N. and Vauthrin, R., 2000, Impact of field parameters on surface multiple attenuation - a 3D example, 62nd Mtg.: Eur. Assn. Geosci. Eng., Session:L0055.

Woodward, M. J., Farmer, P., Nichols, D. and Charles, S., 1998, Automated 3-D tomographic velocity analysis of residual moveout in prestack depth migrated common image point gathers, 68th Ann. Internat. Mtg: Soc. of Expl. Geophys., 1218-1221. 